\title{
THE STRONG LAW OF LARGE NUMBERS FOR MARTINGALES WITH DETERMINISTIC QUADRATIC VARIATION
}

\author{
KACHA DZHAPARIDZE \\ Centre for Mathematics and Computer Science, \\ P.O. Box 4079, 1009 AB Amsterdam, The Netherlands \\ PETER SPREIJ \\ Department of Econometrics, Free University, \\ De Boelelaan 1105, 1081 HV Amsterdam, The Netherlands \\ (Received 1 September 1991; in final form 20 May 1992)
}

\begin{abstract}
The strong law of large numbers is proved for multivariate martingales with deterministic quadratic variation, along the same lines as in Lai, Wei and Robbins (1979), though the setting here is more general.

KEY WORDS Martingale, quadratic variation process, strong law of large numbers, least squares estimation.
\end{abstract}

\section{INTRODUCTION}

1.1 For scalar valued martingales the strong law of large numbers (SLLN) is relatively easily proved: if $M$ is locally square integrable martingale, then $\langle M\rangle_{t}^{-1} M_{t}$ converges a.s. as $t \rightarrow \infty$ and the limit equals zero if $\langle M\rangle_{t} \rightarrow \infty$ a.s. (see Liptser and Shiryayev, 1989, Section 2.6). But in the multivariate case the matter is different due to the possibly complicated dependence structure between the components (see e.g. Christopeit (1986), Lai and Wei (1982), Le Breton and Musiela (1987, 1989), Mel'nikov (1986) and Novikov (1985)). The SLLN in this case refers to $\langle M\rangle_{t}^{-1} \mathbb{M}_{t} \rightarrow 0$ a.s. as $t \rightarrow \infty$, where $\mathbb{M}$ is an $\mathbb{R}^{d}$ valued locally square integrable martingale and $\langle\mathbb{M}\rangle$ is the $\mathbb{R}^{d \times d}$ valued tensor predictable covariation process. The motivation in the above mentioned papers for investigating whether a SLLN holds traditionally stems from (pseudo) least squares estimation.

As is shown in this paper, the problem still has a relatively simple solution under the restriction that the quadratic variation process of the multivariate margingale in question is deterministic.

The first result in this direction has been proved by Lai, Wei and Robbins (1979) in the discrete time setting in a paper on least squares estimation (see also Le Breton and Musiela (1986)). Their proofs heavily depend on the fact that all components are actually transforms of one and the same real valued margingale. Both these limitations are dropped in the present paper. Our approach is much in spirit of Lai, Wei 
and Robbins (1979), and loosely speaking generalizes all the intermediate steps undertaken in it. It turns out that the intermediate results can be presented in a more compact and elegant form; see Sections 3 and 4 below. We want to empasize that there is a good reason for taking up this older method for analysing the behaviour of $\langle\mathbb{M}\rangle^{-1} \mathbb{M}$ instead of what most recent authors do, which is giving bounds for the quadratic form $\mathbb{M}^{\prime}\langle\mathbb{M}\rangle^{-1} \mathbb{M}$ and then applying Schwarz' inequality which finally yields conditions on the growth rates of minimal and maximal eigenvalues of $\langle\mathbb{M}\rangle$. It appears to us that this approach is not suitable to obtain the sharper results of ours in the presence of the restriction that $\langle M\rangle$ is deterministic, whereas the dimension reduction technique of Lai, Wei and Robbins (1979) provides a useful tool to obtain a criterion under which the SLLN holds in terms of an intuitively appealing probabilistic interpretation.

It should be noticed however that unlike the present paper in Lai, Wei and Robbins (1979) the object in question is not necessarily formed by transforming a real valued martingale (but actually any so-called convergence system: see e.g. Chen, Lai and Wei (1981), Lai and Wei (1984); cf. also Solo (1981)), while in Kaufmann (1987) it is a transformation of a real valued martingale which satisfies some moment conditions.

1.2 In Section 2 the main results of this paper are formulated. The calculations presented in Section 3 are then used for proving in Section 5 a key convergence theorem formulated in Section 4. The proof of the main theorem 1 is given in Section 6. Finally, we discuss in Section 7 an application to least squares estimation.

\section{MAIN RESULTS}

2.1 The basic setting is as follows. On a complete filtered probability space $(\Omega, \mathscr{F}$, $\mathbb{F}, P)$ all our stochastic processes are defined. All martingales are understood as being so with respect to the filtration $\mathbb{F}$.

Let $\mathbb{M}: \Omega \times[0, \infty) \rightarrow \mathbb{R}^{d}$ be a martingale. Let $\langle\mathbb{M}\rangle: \Omega \times[0, \infty) \rightarrow \mathbb{R}^{d \times d}$ be its predictable quadratic variation process. So we assume that for all components $m^{i}$ of $\mathbb{M}$ we have that $E\left(m_{t}^{i}\right)^{2}<\infty$, for all $t \geq 0$, that is $\mathbb{M} \in \mathfrak{M}_{d}^{2}$. Moreover, we will assume throughout this paper that the quadratic variation process $\langle\mathbb{M}\rangle$ is deterministic. So for its $i j$-element we have $\langle M\rangle^{i j}=E\left(m^{i} m^{j}\right)$.

It may happen that for some (or all) $t$ the matrix $\langle\mathbb{M}\rangle_{t}$ is singular. Therefore we will consider $\varepsilon I_{d}+\langle M\rangle_{\text {, }}$, where $\varepsilon>0$ and $I$ the identity matrix, and denote it by $\mathbb{A}_{t}$. Let $\mathbb{V}=\left(\varepsilon I_{d}+\langle\mathbb{M}\rangle\right)^{-1}=\mathbb{A}^{-1}$. (It is also possible to study directly $\langle\mathbb{M}\rangle^{+} \mathbb{M}$, where $\langle\mathbb{M}\rangle^{+}$is the Moore-Penrose generalized inverse. This seems however to lead to many technical complications, that are beyond the purpose of this paper; see Dzhaparidze and Spreij (1992) for a related discussion).

2.2 We will be only interested in the limit behaviour of $\mathbb{V}_{t} \mathbb{M}_{t}$ as $t \rightarrow \infty$ and we will show that under suitable assumptions

$$
\mathbb{V}_{t} \mathbb{M}_{t} \rightarrow 0 \text { a.s. }
$$


First we introduce some notations. Let $e_{i}$ be the ith unit vector in $\mathbb{R}^{d}$ and $c_{i t}^{-1}=e_{i}^{\prime} \mathbb{V}_{t} e_{i}$. Let $g:[0, \infty) \rightarrow \mathbb{R}$ be such that the following integral exists

$$
\int_{0}^{\infty}\left(\frac{g(x)}{x}\right)^{2} d x<\infty
$$

Let $D:[0, \infty) \rightarrow \mathbb{R}^{d \times d}$ be such that $D_{t}$ is a diagonal matrix for all $t \geq 0$, with diagonal elements $D_{i t}=g\left(c_{i t}\right)$.

2.3 The main result of this paper is the following

THeOREM 1 Let $g, c, \mathbb{V}$ and $D$ be as defined above. Then

$$
\lim _{t \rightarrow \infty} D_{t} \mathbb{V}_{t} \mathbb{M}_{t} \text { exists and is finite a.s. }
$$

Moreover if $\lim _{t \rightarrow \infty} c_{i t}=\infty$, then $\lim _{t \rightarrow \infty} e_{i}^{\prime} D_{t} \mathbb{V}_{t} \mathbb{M}_{t}=0$ a.s.

The proof of this theorem is presented in Section 5. It involves a series of auxiliary results, which we present after some additional computations.

Remark Suppose one is only interested in a weak law of large numbers, that is $\langle\mathbb{M}\rangle_{t}^{-1} \mathbb{M}_{t} \rightarrow 0$ in probability as $t \rightarrow \infty$ (assume here that $\langle\mathbb{M}\rangle_{t}^{-1}$ exists for $t$ large enough). A sufficient condition is then $\mathbb{E M}_{t}^{\prime}\langle\mathbb{M}\rangle_{t}^{-2} \mathbb{M}_{t} \rightarrow 0$, which is equivalent to $\operatorname{tr}\langle M\rangle_{t}^{-1} \rightarrow 0$ and hence $c_{i t} \rightarrow \infty(i=1, \ldots, d)$. The observation that $c_{i t} \rightarrow \infty$ is sufficient for a weak law to hold, suggests that a condition on the behaviour of the eigenvalues of $\langle M\rangle$ should be superfluous for the SLLN. One of the aims of this paper is to show that this suggestion can be justified. Indeed it follows from Theorem 1 that $\langle\mathbb{M}\rangle_{t}^{-1} \rightarrow 0$ is a sufficient condition for the SLLN to hold, and also gives some information on the rate of convergence in terms of the matrices $D_{t}$.

2.4 Assertion (i) of the following corollary is obvious, and assertion (ii) is proved in Section 7.2 .

\section{COROllary 2}

i) Let $\langle\mathbb{M}\rangle$, be non singular for $t$ large enough. Then the assertion of Theorem 1 remains true if we take $\varepsilon=0$.

ii) Assume $\lim _{t \rightarrow \infty} u^{\prime}\langle\mathbb{M}\rangle_{t} u$ for all $u \in \mathbb{R}^{d}$ is either zero or infinity. Then

$$
\lim _{t \rightarrow \infty} \mathbb{V}_{t} \mathbb{M}_{t}=0 \text { a.s. }
$$

This statement remais valid if $\mathbb{V}$ is substituted by a generalized inverse $\langle\mathbb{M}\rangle^{+}$. 


\section{AUXILIARY ASSERTIONS}

3.1 First we introduce some more notations. Write $\mathbb{M}=\left[\begin{array}{c}m \\ M\end{array}\right]$, where $m \in \mathfrak{M}_{1}^{2}$ and $M \in \mathfrak{M}_{d-1}^{2}$. Surely $m=e_{1}^{\prime} \mathbb{M}$ and $M=J_{d}^{\prime} \mathbb{M}$ with $J_{d}^{\prime}=\left[0, I_{d-1}\right]$. Denote $A=\varepsilon I_{d-1}+$ $\langle M\rangle=J_{d}^{\prime} A J_{d}$ and $V=A^{-1}$.

We repeatedly will use the following identities:

$$
\begin{gathered}
d A V+A_{-} d V=d V A+V_{-} d A=0, \quad d A+A_{-} d V A=d V-V_{-} d A V=0 \\
d A+A d V A=-d A V_{-} \Delta A \geq 0, \quad d V+V d A V=-d V A_{-} \Delta V \leq 0 .
\end{gathered}
$$

We can present $\mathbb{V}$ as follows:

$$
\mathbb{V}=c^{-1} b b^{\prime}+J_{d} \mathbb{V} J_{d}^{\prime} \quad \text { with } \quad b=\left[\begin{array}{c}
1 \\
-V\langle M, m\rangle
\end{array}\right] \text { and } c^{-1}=c_{1}^{-1}=e_{1}^{\prime} \mathbb{V} e_{1}
$$

(Here and elsewhere the time index $t$ will often be omitted). This is easily seen by using the following representation for $\mathbb{A}=\varepsilon I_{d}+\langle\mathbb{M}\rangle$ :

$$
\mathbb{A}=\left[\begin{array}{cc}
1 & \langle m, M\rangle V \\
0 & I_{d-1}
\end{array}\right]\left[\begin{array}{ll}
c & 0 \\
0 & A
\end{array}\right]\left[\begin{array}{cc}
1 & 0 \\
V\langle M, m\rangle & I_{d-1}
\end{array}\right]
$$

where $c=a-\langle m, M\rangle V\langle M, m\rangle$ with $a=\varepsilon+\langle m\rangle$. Observe that

$$
\mathbb{A} b=c e_{1} \quad \text { and } \quad c=\operatorname{det} \mathbb{A} / \operatorname{det} A=b^{\prime} \mathbb{A} b=b^{\prime} A_{1} \quad \text { with } \quad A_{1}=\mathbb{A} e_{1} .
$$

Hence by (3)

$$
\mathbb{V M}=c^{-1} b b^{\prime} \mathbb{M}+\left[\begin{array}{c}
0 \\
V M
\end{array}\right]
$$

and we see that the first component in (6) is equal to $c^{-1} b^{\prime} M$. Therefore it is easily seen that studying of $\mathbb{V} \mathbb{M}$ is equivalent to studying of quantities like $c^{-1} b^{\prime} \mathbb{M}$, since any component of $\mathbb{V M}$ is of this form after a suitable permutation of $\mathbb{M}$ and $\langle\mathbb{M}\rangle$.

3.2 We need the following multivariate version of Theorem 8 in Liptser and Shiryayev (1989), Section 2.2, adapted to the present situation.

Proposition 3 Let $\mathbb{M}$ and $M$ be as above. There exists a predicable $d \times(d-1)$ matrix valued process $\phi$ with the following properties:

i) $\phi d\langle M\rangle=d\langle\mathbb{M}, M\rangle$,

ii) $\phi d\langle M\rangle \phi^{\prime} \leq d\langle\mathbb{M}\rangle$.

The proof proceeds along the same lines as in the univariate case. 
Remark Unlike the last case the process $\phi$ here may be not uniquely determined as, for instance, in the typical case in which $M=v \cdot m$ with a vector valued function $v$ and a sclar valued martingale $m$, because now $d\langle M\rangle_{t} / d\langle m\rangle_{t}=v_{t} v_{t}^{\prime}$ is singular for each $t$. However the martingale $\phi \cdot M$ does not depend on the particular choice of $\phi$. Here and elsewhere below $\cdot$ means stochastic integration.

3.3 The behaviour of $b^{\prime} \mathbb{M}$ will be studied by representing it as

$$
b^{\prime} \mathbb{M}=b^{\prime} \cdot \mathbb{M}+\mathbb{M}_{-}^{\prime} \cdot b
$$

Proposition 4 Let $N=b^{\prime} \cdot M$ and $n=b^{\prime} \phi \cdot M$ with (the integration variable is usually omitted)

$$
\langle N\rangle_{t}=\int_{[0, t]} b^{\prime} d\langle M\rangle b \text { and }\langle n\rangle_{t}=\int_{[0, t]} b^{\prime} \phi d\langle M\rangle \phi^{\prime} b .
$$

Then

i) $\mathbb{M}_{-}^{\prime} \cdot b_{t}=-\int_{[0, t]} M_{-}^{\prime} V_{-} d\langle M\rangle \phi^{\prime} b$.

ii) $\langle N-n, M\rangle=0$.

iii) $\langle N\rangle_{t}-\langle n\rangle_{t}=c_{t}+\int_{[0, t]} b^{\prime} \phi A d V A \phi^{\prime} b$.

iv) $\langle n\rangle_{t} \leq\langle N\rangle_{t} \leq c_{t}, t \geq 0$, and moreover $d\langle n\rangle / d c \leq 1$ and $d\langle N\rangle / d c \leq 1$.

v) $d V_{1} \ll d c^{-1}$ where $V_{1}=\mathbb{V} e_{1}$.

Proof

i) By (2)

$$
d b=\left[\begin{array}{c}
0 \\
-V_{-} d\langle M\rangle \phi^{\prime} b
\end{array}\right]=\left[\begin{array}{c}
0 \\
-V d\langle M\rangle \phi_{-}^{\prime} b_{-}
\end{array}\right] .
$$

Indeed, the second and third equality, for instance, are easily verified as follows:

$$
\begin{aligned}
d(V\langle M, m\rangle) & =V_{-}(d\langle M, m\rangle-d\langle M\rangle V\langle M, m\rangle) \\
& =V_{-}[d\langle M, m\rangle d\langle M\rangle] b=V_{-} d\langle M, \mathbb{M}\rangle b=V_{-} d\langle M\rangle \phi^{\prime} b .
\end{aligned}
$$

Now, (i) follows from (8).

ii) As is easily seen by definition of $\phi$ in Proposition 3, the martingales $N-n$ and $M$ are orthogonal:

$$
\langle N-n, M\rangle=b^{\prime} \cdot\langle\mathbb{M}, M\rangle-b^{\prime} \phi \cdot\langle M\rangle=0 .
$$

iii) By (5) $d c=d\left(b^{\prime} \mathbb{A}\right) e_{1}=b_{-}^{\prime} d\langle\mathbb{M}\rangle e_{1}+d b^{\prime} \mathbb{A} e_{1}$, hence

$$
d c=b_{-}^{\prime} d\langle\mathbb{M}\rangle b
$$


and

$$
\begin{aligned}
\langle N\rangle_{t}-c_{t} & =\sum_{[0, t]} \Delta b^{\prime} \Delta\langle\mathbb{M}\rangle b=\sum_{[0, t]}\left[0-b^{\prime} \Delta\langle\mathbb{M}, M\rangle V_{-}\right] \Delta\langle\mathbb{M}\rangle b \\
& =-\sum_{[0, t]} b^{\prime} \Delta\langle\mathbb{M}, M\rangle V_{-} \Delta\langle M, \mathbb{M}\rangle b=-\sum_{[0, t]} b^{\prime} \phi \Delta\langle M\rangle V_{-} \Delta\langle M\rangle \phi^{\prime} b .
\end{aligned}
$$

This gives (iii), since by (2) we have

$$
\begin{aligned}
\langle n\rangle_{t}+\int_{[0, t]} b^{\prime} \phi A d V A \phi^{\prime} b & =\sum_{[0, t]} b^{\prime} \phi \Delta\langle M\rangle \Delta V A \phi^{\prime} b \\
& =-\sum_{[0, t]} b^{\prime} \phi \Delta\langle M\rangle V_{-} \Delta\langle M\rangle \phi^{\prime} b .
\end{aligned}
$$

iv) Surely, (iii) implies (iv), since the second term on the right hand side of (iii) gives a nonnegative contribution.

v) Observe that $c^{-1}$ is non increasing because $c^{-1}=e_{1}^{\prime} \mathbb{V} e_{1}$ with $\mathbb{V}$ non increasing, since $\langle\mathbb{M}\rangle$ is non decreasing. The equality $V_{1}=c^{-1} b$ follows from the first relation in (5).

For any non negative (measurable) function $q$ we have

$$
0 \leq-\int_{[0, \infty)} q d c^{-1}=-\int_{[0, \infty)} q e_{1}^{\prime} d \mathbb{V} e_{1} \geq \int_{[0, \infty)} q V_{1}^{\prime} d A V_{1}
$$

by (2), so that if $q=0 d c^{-1}$ a.e., then

This means that

$$
\int_{[0, \infty)} q V_{1}^{\prime} d A V_{1}=0
$$

$$
\int_{[0, \infty)} q d \mathbb{A} V_{1}=-\int_{[0, \infty)} q \mathbb{A}-d V_{1}=0, \text { as } \quad \mathbb{A}>0
$$

(see (2)). Hence $q \mathbb{A}_{-}$is $d V_{1}$ a.e. zero on $(0, \infty)$ and so $q$ is a.e. zero on $(0, \infty)$ with respect to $d V_{1}$.

\section{A CONVERGENCE THEOREM}

4.1 Theorem 5 formulated in this section is crucial for studying the behaviour of $\mathrm{M}_{-}^{\prime} \cdot b$.

Let $A:[0, \infty) \rightarrow \mathscr{P}^{d}$ where $\mathscr{P}^{d}$ is the set of non negative definite $(d \times d)$-matrices. Assume that $A_{0}>0$ and that $A$ is non decreasing, so $A_{t} \geq A_{s}$ for $t \geq s$. Since all the $A_{t}$ are invertible, $V_{t}=A_{t}^{-1}$ is well defined for all $t \geq 0$, and for $t>0$ we have $d V=-V d A V_{-}(\operatorname{see}(2))$. 
For $h:[0, \infty) \rightarrow \mathbb{R}^{r}$ we use the following notation $h \in L^{2}([0, \infty), d A)$ if the following integral is well defined and finite:

$$
\int_{[0, \infty)} h^{\prime} d A h
$$

Define similarly $L^{2}((0, \infty), d V)$; observe that $d V_{t} \leq 0$.

THEOREM 5 Let $\mathbb{M}$ be an $\mathbb{R}^{d}$-valued martingale with $\langle\mathbb{M}\rangle_{t}=E\left(\mathbb{M}_{t} \mathbb{M}_{t}^{\prime}\right)<\infty$ for all $t \geq 0$. Let $\mathbb{A}=\varepsilon I+\langle\mathbb{M}\rangle, \mathbb{V}=\mathbb{A}^{-1}$ and $\mathbb{b}:(0, \infty) \rightarrow \mathbb{R}^{d}, \mathbb{h}_{\mathrm{b}} \in L^{2}((0, \infty), d \mathbb{V})$. Then

$$
\lim _{t \rightarrow \infty} \int_{[0, t]} \mathbb{b}^{\prime} d \mathbb{V} \mathbb{M}_{-} \quad \text { exists and is finite a.s. }
$$

4.2 The proof of this theorem is given in Section 5. It is based on a series of technical lemmas which are presented below.

LeMma 6 For a given $h \in L^{2}((0, \infty), d V)$, the function $\tilde{h}:[0, \infty) \rightarrow \mathbb{R}^{d}$ given by

$$
\tilde{h}_{t}=\int_{[t, \infty)} d V h
$$

is well defined, and moreover $\tilde{h} \in L^{2}([0, \infty), d A)$.

Proof We prove the following three facts:

i) $\tilde{h}_{t}^{\prime} A_{t} \tilde{h}_{t}$ is finite for all $t \geq 0$ and tends to zero as $t \rightarrow \infty$, which also shows that $\tilde{h}_{t}$ is well defined for all $t \geq 0$.

ii) $V h \in L^{2}((0, \infty), d A)$

iii) $\hat{h}=\tilde{h}-V h \in L^{2}((0, \infty), d A)$ and $\int_{(0, \infty)} \hat{h}^{\prime} d A \hat{h}=-\int_{(0, \infty)} h^{\prime} d V h-\tilde{h}_{0}^{\prime} A_{0} \tilde{h}_{0}$.

Observe that the last fact means that $\tilde{h} \in L^{2}([0, \infty), d A)$, since

$$
\int_{[0, \infty)} \tilde{h}^{\prime} d A \tilde{h}=\int_{(0, \infty)} \tilde{h}^{\prime} d A \tilde{h}+\tilde{h}_{0}^{\prime} A_{0} \tilde{h}_{0} \quad \text { with the convention } A_{0-}=0
$$

i) Denote by $R$ the matrix such that $A=R^{2}$ and $R=R^{\prime}$. Taking into consideration that $\lim V_{t}=V_{\infty}$ exists and is positive semi-definite, we get (i) due to the following consequence of Schwartz' inequality:

$$
\begin{aligned}
\tilde{h}_{t}^{\prime} A_{t} \tilde{h}_{t} & =\sum_{i}\left(e_{i}^{\prime} R_{t} \tilde{h}_{t}\right)^{2}=\sum_{i}\left[\int_{(t, \infty)} e_{i}^{\prime} R_{t} d V_{s} h_{s}\right]^{2} \leq \sum_{i} e_{i}^{\prime} R_{t} \int_{(t, \infty)} d V_{s} R_{t} e_{i} \int_{(t, \infty)} h^{\prime} d V h \\
& =\sum_{i} e_{i}^{\prime} R_{t}\left(V_{\infty}-V_{t}\right) R_{t} e_{i} \int_{(t, \infty)} h^{\prime} d V h \leq-\sum_{i} e_{i}^{\prime} R_{t} V_{t} R_{t} e_{i} \int_{(t, \infty)} h^{\prime} d V h \\
& =-d \int_{(t, \infty)} h^{\prime} d V h,
\end{aligned}
$$

since $\sum_{i} e_{t}^{\prime} R_{t} V_{t} R_{t} e_{i}=d$. 
ii) On $(0, \infty)$ the identities (2) are valid, so that (ii) is implied by $d V+V d A V \leq 0$.

iii) Along with the identities (2), we have $d \tilde{h}=d V h$ on $(0, \infty)$. Now, by

$\hat{h}-\tilde{h}_{-}=-V_{-} h$ and $\tilde{h}^{\prime} d(A \tilde{h})=\tilde{h}^{\prime} A_{-} d V h+\tilde{h}^{\prime} d A \tilde{h}=\tilde{h}^{\prime} d A \tilde{h}-\tilde{h}^{\prime} d A V h=\tilde{h}^{\prime} d A \hat{h}$

we get

$\hat{h}^{\prime} d A \hat{h}-d\left(\tilde{h}^{\prime} A \tilde{h}\right)=\left(\hat{h}^{\prime}-\tilde{h}^{\prime}\right) d A \hat{h}-h^{\prime} d V A_{-} \tilde{h}_{-}=h^{\prime} V d A\left(\tilde{h}_{-}-\hat{h}\right)=-h^{\prime} d V h$.

Hence

$$
d\left(\tilde{h}^{\prime} A \tilde{h}\right)=\hat{h}^{\prime} d A \hat{h}+h^{\prime} d V h \text { and }-\int_{[0, \infty)} \hat{h}^{\prime} d A \hat{h}=\int_{[0, \infty)} h^{\prime} d V h+\tilde{h}_{0}^{\prime} A_{0} \tilde{h}_{0},
$$

where we have used (i).

LEMMA 7 Let $m$ be a real valued square integrable martingale. Let $A$ be an increasing function with $A_{0}>0$ such that $\langle m\rangle \ll A$ and $d\langle m\rangle / d A$ is bounded. Assume $h \in L^{2}(d V)$ where $V=1 / A$. Then

$$
\lim _{t \rightarrow \infty} \int_{[0, t]} h m_{-} d V \text { exists and is finite a.s. }
$$

Proof Integrating by parts we get $\int_{[0, t]} h m_{-} d V=\tilde{h}_{t} m_{t}-\int_{[0, t]} \tilde{h} d m$ where $\tilde{h}$ is given by (10). Then $\tilde{h} \in L^{2}([0, \infty), d A)$ in view of Lemma 5 . Let now

$$
\tilde{m}=\int_{[0, t]} \tilde{h} d m \text { with } E \tilde{m}_{t}^{2}=\int_{[0, t]} \tilde{h}^{2} d\langle m\rangle=\int_{[0, t]} \tilde{h}^{2} \frac{d\langle m\rangle}{d A} d A,
$$

which is bounded in $t$. Hence $\lim _{t \rightarrow \infty} \tilde{m}_{t}$ exists and is finite a.s. Surely also $\int_{[0, t]} k d m$ has a limit a.s. where $k_{t}=-\int_{(t, \infty)}|h| d V$. Then Kronecker's lemma for martingales (see Lipster and Shiryayev (1989), Section 2.6) applies, since $\left|h_{t}\right|$ decreases to zero, which yields $\left|\tilde{h}_{t}\right| m_{t} \rightarrow 0$ a.s. and hence $\left|\tilde{h}_{t} m_{t}\right| \rightarrow 0$ a.s.

4.3 We want to emphasize here that in this lemma it is important that $h$ and $V$ are deterministic, because now $\tilde{h}$ is also deterministic and therefore $\tilde{m}$ in Section 4.2 is a convergent martingale. If we would have started with predictable processes $h$ and $V$, it would be not have been possible to define, as we did above, a martingale like $\tilde{m}$.

It is indeed Lemma 7, and its generalization Theorem 5, that has no counterpart if one wants to treat only predictable quadratic variation processes. Therefore we want to stress that it is at this point that we obtain sharper results then, for instance, in Christopeit (1986), Lai and Wei (1982), Le Breton and Musiela (1987, 1989), Mel'nikov (1986) or Novikov (1985). 


\section{PROOF OF THEOREM 5}

5.1 We use induction with respect to the dimension $d$ of the space where $M$ takes its values. Clearly for $d=1$ the theorem reduces to Lemma 6. So assume the theorem holds for $d-1$. As in Section 3 we write $\mathbb{M}=\left[\begin{array}{l}m \\ M\end{array}\right]$, preserving all the notations introduced there. Using (6) and the relation $d \mathbb{V}=-\mathbb{V} d\langle\mathbb{M}\rangle \mathbb{V}$ - (cf. (2)) we split the integral in question in two terms

$$
\int_{[0, t]} \mathbb{h}^{\prime} d \mathbb{V} \mathbb{M}_{-}=I_{1}(t)+I_{2}(t)
$$

where

$$
I_{1}(t)=\int_{[0, t]} \ln { }^{\prime} \mathbb{V} d\langle\mathbb{M}\rangle\left[\begin{array}{c}
0 \\
V_{-} M_{-}
\end{array}\right]=\int_{[0, t]} \ln \mathbb{V} d\langle\mathbb{M}, M\rangle V_{-} M_{-}=\int_{[0, t]} h^{\prime} d V M_{-}
$$

with $h=A \phi^{\prime} \mathbb{V} \mathfrak{h}$ and $\phi$ defined by $d\langle\mathbb{M}, M\rangle=\phi d\langle M\rangle$ as in Proposition 3, and

$$
I_{2}(t)=\int_{[0, t]} c_{-}^{-1} \mathfrak{b}^{\prime} \mathbb{V} d\langle\mathbb{M}\rangle \mathfrak{b}_{-} \mathfrak{b}_{-}^{\prime} \mathbb{M}_{-}=-\int_{[0, t]} \mathbb{b}^{\prime} d V_{1} \mathfrak{b}_{-}^{\prime} \mathbb{M}_{-}
$$

(see Proposition $4(\mathrm{v})$ ), since $d V_{1}=-\mathbb{V} d\langle M\rangle V_{1}$ - by (2).

5.2 We will show that $h \in L^{2}(d V)$ as $t_{0} \in L^{2}(d \bigvee)$ by assumption, and this will imply that $I_{1}(t)$ has a limit a.s. as $t \rightarrow \infty$, that is

$$
-\int_{[0, \infty)} h^{\prime} d V h<\infty \Rightarrow\left|\int_{[0, \infty)} h^{\prime} d V M_{-}\right|<\infty \text { a.s. }
$$

since by the induction hypothesis we have assumed that the assertion of the theorem holds for $d-1$. In fact, by (2) and Proposition 3 (ii)

$$
\begin{aligned}
-\int_{[0, \infty)} h^{\prime} d V h & \leq-\int_{[0, \infty)} \mathbb{h}^{\prime} \mathbb{V} \phi A d V A \phi^{\prime} \mathbb{V} \mathfrak{h} \\
& \leq \int_{[0, \infty)} \mathbb{h}^{\prime} \mathbb{V} \phi d A \phi^{\prime} \mathbb{V} \mathbb{h} \leq-\int_{[0, \infty)} \mathbb{h}^{\prime} d \mathbb{V} \mathbb{h} .
\end{aligned}
$$

5.3 Next we direct our attention towards $I_{2}(t)$. We write $I_{2}(t)=I_{3}(t)+I_{4}(t)$ with

$$
I_{3}(t)=\int_{[0, t]} \mathbb{h}^{\prime} d V_{1} N_{-}=\int_{[0, t]} \mathbb{h}^{\prime} \frac{d V_{1}}{d \gamma} N_{-} d \gamma \quad \text { and } \quad I_{4}(t)=\int_{[0, t]} \mathbb{h}^{\prime} d V_{1}\left(\mathbb{M}_{-}^{\prime} \cdot b\right)_{-}
$$

where $\gamma=-c^{-1}$ (see Proposition $\left.4(\mathrm{v})\right)$. 
Since $d\langle\mathbb{N}\rangle / d \gamma \leq 1$ by Proposition 4 (iv), $I_{3}(t)$ converges by Lemma 7, since

$$
\int_{[0, \infty)}\left(\mathbb{h}^{\prime} \frac{d V_{1}}{d \gamma}\right)^{2} d \gamma \leq-\int_{[0, \infty)} \mathbb{h}^{\prime} \frac{d \mathbb{V}}{d \tau} \mathbb{h} d \tau=-\int_{[0, \infty)} \mathbb{h}^{\prime} d \mathbb{V} \mathfrak{h}<\infty
$$

with $\tau=\operatorname{tr} \mathbb{V}$ (so that $d \mathbb{V}$ is dominated by $d \tau$ ). We have the second inequality by assumption, and the first by the following consequence of Schwartz' inequality:

$$
\left(\frac{d \gamma}{d \tau}\right)^{2}\left(\mathbb{h}^{\prime} \frac{d V_{1}}{d \gamma}\right)^{2}=\left(\mathbb{h}^{\prime} \frac{d V_{1}}{d \tau}\right)^{2} \leq-\mathfrak{h}^{\prime} \frac{d \mathbb{V}}{d \tau} \operatorname{\Pi n} \frac{d \gamma}{d \tau}
$$

5.4 The next term that we have to consider is $I_{4}(t)$. Introduce

$$
p_{t}=\int_{(t, \infty)} h^{\prime} d V_{1} \text {. }
$$

Integrating by parts we get

$$
I_{4}(t)=p_{t} \int_{[0, t]} b^{\prime} \phi A d V M_{-}-\int_{[0, t]} p b^{\prime} \phi A d V M_{-}
$$

by (2) and Proposition 4 (i). Again, we will show by the induction hypothesis that the second term on the left hand side of (13) has a limit as $t \rightarrow \infty$ a.s., that is by checking that

$$
-\int_{[0, \infty)} p^{2} b^{\prime} \phi A d V A \phi^{\prime} b \leq \int_{[0, \infty)} p^{2} d c<\infty
$$

The first inequality follows from Proposition 4 (iii), and second from the fact that $p \in L^{2}(d \gamma)$ with $\gamma=-1 / c$, which is verified as follows: in view of Proposition $4(\mathrm{v})$, write

$$
p_{t}=\int_{(t, \infty)} \ln ^{\prime} \frac{d V_{1}}{d \gamma} d \gamma
$$

and then apply Lemma 6 (scalar case). Hence, the second term in (13) converges a.s. as $t \rightarrow \infty$. Of course, if in this term we replace $p_{t}$ by

$$
\int_{(t, \infty)}\left|\mathbb{h}^{\prime} \frac{d V_{1}}{d \gamma}\right| d \gamma
$$

then we still have that the a.s. limit exists as $t \rightarrow \infty$. Using Kronecker's lemma again, we get from (13) that $I_{4}(t)$ converges a.s. as $t \rightarrow \infty$. This concludes the proof of Theorem 5 . 


\section{PROOF OF THEOREM 1}

6.1 It is sufficient to look at the first component of $D \mathbb{V M}$ which, in the notations of Sections 2 and 3, can be written as follows:

$$
c^{-1} g(c) N+c^{-1} g(c) \mathbb{M}_{-}^{\prime} \cdot b .
$$

If $c$ is bounded, so is $\langle N\rangle$ (see Proposition 4 (iv)) and then both $\lim _{t \rightarrow \infty} c_{t}^{-1} g\left(c_{t}\right)$ and $\lim _{t \rightarrow \infty} N_{t}$ are finite a.s. If $c_{t} \rightarrow \infty$, then $c_{t}^{-1} g\left(c_{t}\right) N_{t}$ still has a finite limit which equals zero as

$$
\int_{[0, \infty)}\left(c^{-1} g(c)\right)^{2} d\langle N\rangle \leq \int_{[0, \infty)}\left(c^{-1} g(c)\right)^{2} d c<\infty
$$

by (1) and Proposition 4 (iv).

6.2 Next we look at the second term in (14). Consider first

$$
\int_{[0, t]} c^{-1} g(c) \mathbb{M}_{-}^{\prime} d b=-\int_{[0, t]} c^{-1} g(c) M_{-}^{\prime} V_{-} d\langle M\rangle \phi^{\prime} b=\int_{[0, t]} c^{-1} g(c) M_{-}^{\prime} d V A \phi^{\prime} b
$$

(see (2) and Proposition 4 (i)). According to Theorem 5 this expression converges since

$$
-\int_{[0, \infty)}\left(c^{-1} g(c)\right)^{2} b^{\prime} \phi A d V A \phi^{\prime} b \leq \int_{[0, \infty)}\left(c^{-1} g(c)\right)^{2} d c<\infty
$$

by (1) and Proposition 4 (iii) and (iv).

If $c_{t}$ converges to a finite limit, then it is seen, in a similar manner as above, that $\left(\mathrm{M}_{-}^{\prime} \cdot b\right)_{t}$ has a finite limit a.s. as $t \rightarrow \infty$. If $c_{t} \rightarrow \infty$, then Kronecker's lemma gives that the second term in (14) tends to zero. Theorem 1 is proved.

\section{ADDITIONAL REMARKS. APPLICATION TO LEAST SQUARES ESTIMATION}

7.1 It may happen that $\lim _{t \rightarrow \infty} \mathbb{V}_{t} \mathbb{M}_{t}=0$ a.s. even if the functions $c_{i t}$ remain bounded. Consider for instance the following example.

Example Let $w$ be a standard Brownian motion, and $v \in \mathbb{R}^{d}$. Let $\mathbb{M}_{t}=v w_{t}$ with $\langle M\rangle_{t}=v v^{\prime} t$. Consider

$$
\left.\mathbb{V}_{t}=\left(\varepsilon I_{d}+M\right\rangle_{t}\right)^{-1}=\varepsilon^{-1}\left(I_{d}-\left(\varepsilon+v^{\prime} v t\right)^{-1} v v^{\prime} t\right) .
$$

We see that $c_{i t}^{-1}=\varepsilon^{-1}\left(\varepsilon+v^{\prime} v t\right)^{-1}\left(v^{\prime} v-v_{i}^{2}\right) t$, where $v_{i}$ is the $i$-th component of $v$, tends to $\varepsilon^{-1}\left(v^{\prime} v-v_{i}^{2}\right) / v^{\prime} v$ which is in general larger than zero. However

$$
\lim _{t \rightarrow \infty} \mathbb{V}_{t} \mathbb{M}_{t}=\lim _{t \rightarrow \infty} v\left(\varepsilon+v^{\prime} v t\right)^{-1} w_{t}=0 \text { a.s. }
$$


Observe that in this example $\langle\mathbb{M}\rangle_{t}$ is singular for all $t$. Careful inspection of this example leads to assertion (ii) of Corollary 2 .

7.2 This assertion will be proved here. Notice first that rank $\langle\mathbb{M}\rangle_{t}$ is increasing. Assume $\lim _{t \rightarrow \infty} \operatorname{rank}\langle\mathbb{M}\rangle_{t}=k\left\langle d\right.$. Then there is $t_{k}>0$ such that $\operatorname{ran} k\langle\mathbb{M}\rangle_{t}=k$ for $t \geq t_{k}$. Assume below that $t \geq t_{k}$. Write $\langle\mathbb{M}\rangle_{t}=r_{t} r_{t}^{\prime}$, with rank $r_{t}=k$. Then

$$
\mathbb{V}_{t}=\varepsilon^{-1}-\varepsilon^{-1} r_{t}\left(I_{d}+\varepsilon^{-1} r_{t}^{\prime} r_{t}\right)^{-1} r_{t}^{\prime} \varepsilon^{-1}
$$

Since there exist a constant matrix $K$ and a martingale $Y_{t}$ with values in $\mathbb{R}^{k}$ such that $\mathbb{M}_{t}=K Y_{t}$, and an invertible matrix $\rho_{t}$ such that $r_{t}=K \rho_{t}$ and $\rho_{t} \rho_{t}^{\prime}=\langle Y\rangle_{t}$ (this claim is proved below), we have

$$
\mathbb{V} M=\mathbb{V} K Y=\mathbb{V} r \rho^{-1} Y=\varepsilon^{-1} r\left(I_{d}+\varepsilon^{-1} r^{\prime} r\right)^{-1} \rho^{-1} Y=r\left(\varepsilon I_{d}+r^{\prime} r\right)^{-1} \rho^{\prime}\langle Y\rangle^{-1} Y .
$$

Use now $r\left(\varepsilon I_{d}+r^{\prime} r\right)^{-1} \rho^{\prime}=\left(I_{d}-\varepsilon \mathbb{V}\right)\left(K^{+}\right)^{\prime}$ where $K^{+}$is a left inverse of $K$. Since the limit of $\mathbb{V}_{t}$ exists as $t \rightarrow \infty$ and $\langle Y\rangle_{t}^{-1} Y_{t}$ tends to zero by Corollary 2 (i), we have $\lim _{t \rightarrow \infty} \mathbb{V}_{t} \mathbb{M}_{t}=0$ a.s.

In conclusion we prove the above claim in italics as follows. In view of the fact that not only $\operatorname{rank}\langle\mathrm{M}\rangle_{t}$ remains constant but also $\operatorname{Im}\langle\mathbb{M}\rangle_{t}=\operatorname{Im} r_{t}$, take now $k$ vectors $\kappa_{1}, \ldots, \kappa_{k} \in \mathbb{R}^{d}$ such that $\operatorname{Im} r_{t}=\operatorname{Im} K$ with $K=\left[\kappa_{1}, \ldots, \kappa_{\kappa}\right]$. Then there exists an invertible matrix $\rho_{t}$ such that $r_{t}=K \rho_{t}$. Define now $Y_{t}=K^{+} \mathbb{M}_{t}$. Then $\mathbb{M}_{t}=K Y_{t}$ a.s. for all $t$. Indeed it is easily verified that $\langle\mathbb{M}-K Y\rangle \equiv 0$, and this proves the claim.

Observe that $\rho_{t} \rho_{t}^{\prime}=\langle Y\rangle_{t}$ and $\langle Y\rangle_{t} \rightarrow \infty$. Indeed for a $v \in \mathbb{R}^{k}, v \neq 0$ there exists $u \in \mathbb{R}^{d}$ such that $v=K^{\prime} u$, since $K^{\prime}$ has a full row rank. Then $v^{\prime}\langle Y\rangle_{t} v=u^{\prime}\langle\mathbb{M}\rangle_{t} u$. If this remains zero, then $u \in \operatorname{Ker}\langle M\rangle_{t}$ for all $t \geq t_{k}$. Hence $u \in \operatorname{Ker} K^{\prime}$, but this contradicts $v \neq 0$. Hence $v^{\prime}\langle Y\rangle_{t} v \rightarrow \infty$.

7.3 As an application we treat least squares estimation for linear models. In many instances it is possible to transform the observations in such a way that we may assume that we observe $x_{s}=\langle m\rangle_{s} \theta+m_{s}$ on $0 \leq s \leq t$, where $m$ is an $\mathbb{R}^{d}$ valued square integrable martingale and $\theta$ an unknown $d$-dimensional parameter. (For example in case of the familiar model $y_{s}=a_{s}^{\prime} \theta+\varepsilon_{s}, s=1, \ldots, t$ one may define $\left.x_{s}=a_{1} y_{1}+\cdots+a_{s} y_{s}\right)$.

The least squares estimator for $\theta$ by definition then minimizes

$$
\left(x_{t}-\langle m\rangle_{t} \theta\right)\langle m\rangle_{t}^{+}\left(x_{t}-\langle m\rangle_{t} \theta\right)
$$

where $\langle m\rangle_{t}^{+}$is a generalized inverse of $\langle m\rangle_{t}$. The set of least square estimators $\theta_{t}$ is given by $\left\{\langle m\rangle_{t}^{+} x_{t}+K \mid K \in \operatorname{Ker}\langle m\rangle_{t}\right\}$. If $\langle m\rangle_{t}$ eventually becomes non singular, then $\theta_{t}-\theta=\langle m\rangle_{t}^{-1} m_{t}$ and Corollary 2 (i) applies. Otherwise let $K$ be as in Section 7.2. Preserving then the notations used there we have

$$
K^{\prime}\left(\theta_{t}-\theta\right)=K^{\prime}\langle m\rangle_{t}^{+} m_{t}=\left(K K^{+}\right)^{\prime}\langle Y\rangle_{t}^{-1} K_{t}^{+} m_{t}=\langle Y\rangle_{t}^{-1} Y_{t} \rightarrow 0 \text { a.s. }
$$

whenever $\langle Y\rangle_{t}^{-1} \rightarrow 0$. So we obtain that if $u^{\prime}\langle m\rangle_{t} u$ either tends to infinity for $t \rightarrow \infty$ or remains zero for all $t$, then a.s. $\lim _{t \rightarrow \infty} \theta_{t}-\theta$ belongs to $\lim _{t \rightarrow \infty} \operatorname{Ker}\langle m\rangle_{t}$. 


\section{References}

Breton A. Le and Musiela M. (1986), Ordre de convergence d'estimateurs des moindres carres en regression lineaire multiple. C. R. Acad. Sc. Paris, t. 302, Serie I, n ${ }^{\circ} 8,333-336$.

Breton A. Le and Musiela M. (1987), Laws of large numbers for semimartingales with applications to stochastic regression. Preprint.

Breton A. Le and Musiela M. (1989), Order of convergence of regression parameter estimates in models with infinite variance. J. Mult. Analysis, 31, 59-62.

Christopeit N. (1986), Quasi-least-squares estimation in semimartingale regression models. Stochastics 16, 255-278.

Dzhaparidze K. and Spreij P. (1992). On correlation calculus for multivariante martingales. Stochastic Processes and Appl. (to appear).

Gui-Jing Chen, Lai T. L., and Wei C. Z., (1981), Convergent systems and strong consistency of least squares estimates in regression models. J. Multivariate Analysis 11, 319-333.

Kaufmann H. (1987), On the strong law of large numbers for multiva-iate margingales. Stochastic Processes and Appl. 26, 73-85.

Lai T. L., Robbins H., and Wei C. Z., (1979), Strong consistency of least squares estimates in multiple regression. J. Multivariate Analysis 9, 343-361.

Lai T. L. and Wei C. Z. (1982), Least squares estimates in stochastic regression models with applications to identification and control of dynamic systems. Ann. Statist. 10, 154-166.

Lai T. L. and Wei C. Z. (1984), Moment inequalities with applications to regression and time series models, in Inequalities in Statistics and Probability. IMS Lecture Notes-Monograph Series 5, 163-172.

Liptser R. Sh. and Shiryayev A. N. (1989), Theory of Martingales. Dordrecht, Kluwer Academic Publ. Dordrecht, The Netherlands.

Mel'nikov A. V. (1986), The law of large numbers for multidimensional martingales. Soviet Math. Dokl. 33, 131-135.

Novikov A. A. (1985), Consistency of least squares estimates in regression models with martingale errors, in Statistics and Control of Stochastic Processes. Eds. N. V. Krilov et al. Berlin, Springer, 389-409.

Solo V. (1981), Strong consistency of least squares estimators in regression with correlated distrubances. Ann. Statist. 9, 689-693. 